\title{
A delay timer for presenting auditory and visual probes
}

\author{
GARY E. RANEY and DENNIS SHUMAN \\ University of Florida, Gainesuille, Florida
}

\begin{abstract}
A circuit designed to present auditory or visual probes is described. The circuit allows the probes to be delayed by a specified amount of time, and the volume (for auditory probes) or intensity (for visual probes) may also be adjusted. The circuit may be triggered by any device capable of emitting an electronic pulse-such as a computer or a tachistoscope.
\end{abstract}

Many researchers use a subject's response to a secondary probe stimulus as a measure of performance or cognitive activity (see Kerr, 1973, for a review). In this paradigm, a probe, which is usually a short-duration (typically $100 \mathrm{msec}$ or less) auditory or visual stimulus, is embedded in a primary task. The subject's response to the probe is used as a measure of the cognitive demands of the primary task. For example, Inhoff and Flemming (1989) found that when a visual or auditory probe was presented while people read, probe-detection times (i.e., reaction times) were longer for those who were reading difficult text than for those reading easier text. Wickens, Kramer, Vanasse, and Donchin (1983) found that eventrelated brain potential responses to auditory and visual probes decreased as the difficulty of the primary task increased. Both of these studies demonstrate the usefulness of secondary probe methodologies.

Prior to the widespread use of microcomputers as primary data-collection devices, the hardware implementation of such probes was often system- or project-specific. Computers can be programmed to present such probes, but it is not always easy to control the timing and presentation of a probe precisely. The programming required can be complex. (See Brysbaert, Bovens, D'Ydewalle, \& Van Calster, 1989, for an example of the complexity of timing routines using Turbo Pascal.) In this paper, we describe a simple circuit for presenting auditory and visual probes; it can be used with a wide variety of experimental laboratory systems.

The circuit generates probes of a fixed duration, and it allows the probes' onsets to be delayed by specified amounts of time. The circuit has three primary benefits: First, it may be used with any device capable of emitting an electronic pulse to act as a trigger (e.g., a computer, a tachistoscope, or any other special-purpose digital system). Second, this system can present probes without the use of complex timing routines within a computer pro-

\footnotetext{
We wish to thank Jill Raney and Ira Fischler for their helpful comments and suggestions. Correspondence may be addressed to Gary E. Raney, Department of Psychology, University of Florida, Gainesville, FL 32611-2065.
}

gram. Third, once the delay control is calibrated, the probe delay interval can be increased or decreased quickly and without any modification of software.

\section{Circuit Description}

The circuit contains an operational amplifier and three timer ICs (integrated circuits), and it requires a $\pm 12 \mathrm{~V}$ power supply (see Figure 1). A parts list for the circuit is provided in Table 1 . The amplifier (Type 741), configured as a differential amplifier, allows the circuit to be triggered by a signal even if it is not referenced to ground (such as the signal across the internal speaker of an IBM AT computer). The amplifier has a gain factor of 10 and is used to increase the input signal to a level suitable for triggering the first timer IC.

The first timer IC (Type 555) is configured as a monostable or one-shot-that is, upon being triggered, it produces a single, fixed-duration pulse-and it produces the probe-onset delay period. The trailing edge of this pulse triggers the next IC in the circuit. Connection of the output of the first timer to an oscilloscope allows measurement of the delay interval. A precision digital readout potentiometer is used to control the duration of the delay. The maximum delay is a function of the maximum value of the potentiometer and the capacitor between pin 7 and ground on the timer. Increasing the value of the capacitor increases the maximum delay. As configured, the maximum delay is $2,000 \mathrm{msec}$, and the minimum delay is $0 \mathrm{msec}$.

The second timer IC (Type 555) is also configured as a one-shot. Output from this timer is a positive pulse, the duration of which determines the duration of the probe. Probe duration can be altered by changing the resistor between pin 7 and $+12 \mathrm{~V}$. The duration of the probe may be reduced or increased by using a smaller or a larger value resistor. As configured, probe duration is $100 \mathrm{msec}$. The output of this IC may also be used to drive a visual probe, such as a light-emitting diode (LED). A variable resistor is placed in the circuit to control the intensity of the LED output.

The third timer IC (Type 555) is configured as an oscillator. The frequency of the output is in part controlled 


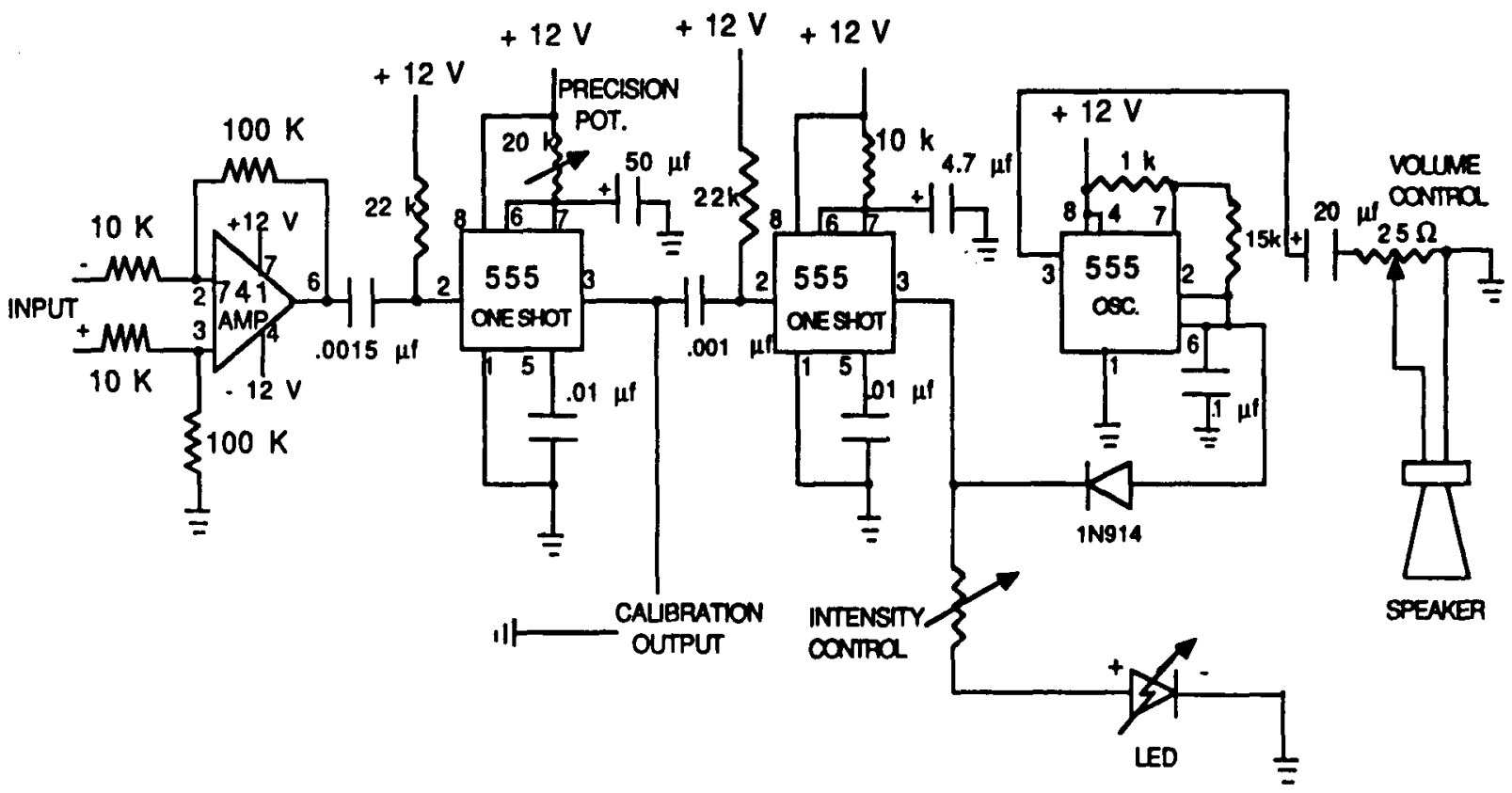

Figure 1. Circuit diagram.

by the resistor between pin 2 and pin 7 . Using a larger value resistor at this point lowers the frequency of the probe, and lower values increase the frequency. As configured, the output from this IC generates a $100-\mathrm{msec}$, $1000-\mathrm{Hz}$ tone when played through a speaker. Probe duration is controlled by the second timer's output, which turns the oscillator on and off by means of the 1N914 diode. The intensity level of the tone is controlled by a potentiometer.

\section{Calibration and Use of the Circuit}

Accurate calibration of the probe delay interval can be achieved by connecting the calibration output from the first timer IC to an oscilloscope (see Figure 1). In our laboratory, the calibration signal is input to a digital oscilloscope. By marking the starting and end points of the calibration signal, one can determine the duration of the probe delay with millisecond accuracy. The temporal resolution for adjusting the delay interval is a factor of the delay range and the resolution of the potentiometer readout used to control the delay. For example, the precision potentiometer has 2,000 divisions ( 0 to 999 , with half steps). With a 2,000-msec delay range, and 2,000 divisions on the potentiometer, the delay can be changed in 1 -msec increments. Once a particular delay interval has been calibrated, one can use the potentiometer's readout to return to that delay with an accuracy of $1 \mathrm{msec}$. Thus we have not depended on the linearity of the potentiometer for determining delay intervals.

The system can be triggered with any electronic pulse from an external device. For example, we have connected the circuit across the computer's speaker. When it is desirable to generate a probe during an experimental trial, the speaker is simply turned on for a very short interval (e.g., $1 \mathrm{msec}$ ) at the onset of the trial. The programming is very simple. For example, in Turbo Pascal, executing the SOUND command followed by the NOSOUND command sends a very short pulse to the speaker, which triggers the circuit. The only requirement of the trigger pulse is that its duration not exceed the duration of the probe delay interval.

The circuit may be used in conjunction with any device capable of generating an electronic pulse to trigger the system. The circuit is ideally suited to conditions that re-

Table 1

Parts List for Constructing the Probe Circuit

\begin{tabular}{llc}
$\begin{array}{c}\text { Catalog } \\
\text { Number }\end{array}$ & \multicolumn{1}{c}{ Description } & Quantity \\
\hline $272-312^{*}$ & 1K resistor & 1 \\
(package & 10K resistor & 3 \\
contains a & 15K resistor & 1 \\
variety of & 22K resistor & 2 \\
resistors) & 100K resistor & 2 \\
$272-802^{*}$ & .0015 uf electrolytic capacitor & 1 \\
(package & .001 uf electrolytic capacitor & 1 \\
contains a & .01 uf electrolytic capacitor & 2 \\
variety of & .1 uf electrolytic capacitor & 1 \\
capacitors) & 4.7 uf electrolytic capacitor & 1 \\
& 20 uf electrolytic capacitor & 1 \\
& 50 uf electrolytic capacitor & 1 \\
$276-1122^{*}$ & 1N914 diode & 1 \\
$276-1723^{*}$ & 555 timer IC & 3 \\
$276-007^{*}$ & 741 amplifier IC & 1 \\
$276-041^{*}$ & light emitting diode & 1 \\
$40-246^{*}$ & speaker (2.25") & 2 \\
$271-265^{*}$ & volume/intensity control & 1 \\
$522-2444 \dagger$ & digital readout potentiometer & \\
\hline
\end{tabular}

*Catalog numbers are for Radio Shack electronics stores. †Catalog number is for Allied Electronics Inc. 
quire a probe of constant duration and constant delay across a set of trials. The simplicity, low cost, and ease and speed of delay adjustment provide a useful alternative to complicated programs and expensive equipment.

\section{REFERENCES}

Brysbaert, M., Bovens, N., D'Ydewalle, G., \& Van Calster, J. (1989). Turbo Pascal timing routines for the IBM microcomputer family. Behavior Research Methods, Instruments, \& Computers, 21, 73-83.
Inhoff, A. W., Flemming, K. (1989). Probe-detection times during the reading of easy and difficult text. Journal of Experimental Psychology: Learning, Memory, \& Cognition, 15, 339-351.

KeRR, B. (1973). Processing demands during mental operations. Memory \& Cognition, 4, 401-412.

Wickens, C., Kramer, A., Vanasse, L., Donchin, E. (1983). Performance of concurrent tasks: A psychophysiological analysis of the reciprocity of information-processing resources. Science, 221, 1080-1081.

(Manuscript received November 3, 1989; revision accepted for publication November 9, 1989.) 